\title{
Distribution of HPV genotypes in 282 women with cervical lesions: evidence for three categories of intraepithelial lesions based on morphology and HPV type
}

\author{
Rosemary E Zuna ${ }^{1}$, Richard A Allen ${ }^{1}$, William E Moore ${ }^{2}$, You Lu ${ }^{1}$, Rubina Mattu ${ }^{1}$ and \\ S Terence Dunn ${ }^{1}$ \\ ${ }^{1}$ Department of Pathology, University of Oklahoma Health Sciences Center, Oklahoma City, OK, USA \\ and ${ }^{2}$ University of Oklahoma Prevention Research Center, Oklahoma City, OK, USA
}

\begin{abstract}
Previously we found differences in the distribution of the individual human papillomavirus types in cervical cancers and high-grade squamous intraepithelial lesions. This suggested that there were differences in risk for progression of high-grade squamous intraepithelial lesions that were related to human papillomavirus type within the category of oncogenic genotypes. In this work, we add additional cases including low-grade squamous intraepithelial lesions. ThinPrep ${ }^{\circledR}$ samples from 282 squamous intraepithelial lesions and invasive cervical cancers were categorized morphologically by consensus interpretation and genotyped for 27 individual human papillomavirus types by polymerase chain reaction-based reverse line blot analysis using PGMY09/ PGMY11 consensus primers for the L1 open reading frame. The 27 human papillomavirus types were divided into three categories: high risk $16,18,31,45$; intermediate risk 33, 35, 39, 51, 52, 56, 58, 59, 68, 73, 82, 83; and low risk: $6,11,26,40,42,53,54,55,57,66$, and 84 . Of the 282 cases of cancer and squamous intraepithelial lesions, $95.7 \%$ were positive for one or more of 27 human papillomavirus types and $38.7 \%$ had two or more genotypes. Three major categories of squamous intraepithelial lesions were identified based upon the combination of consensus diagnosis and human papillomavirus category: (1) high-grade squamous intraepithelial lesions associated with high-risk human papillomavirus types that appear to be at increased risk for progression to carcinoma; (2) squamous intraepithelial lesions (typically low-grade intraepithelial lesions and high-grade lesions consistent with moderate dysplasia) associated with intermediate risk human papillomavirus types with limited or indeterminate risk for progression; (3) low-grade squamous intraepithelial lesions associated with low-risk human papillomavirus types with little or no risk for progression. Only a subset of human papillomavirus genotypes commonly considered to be oncogenic were closely associated with invasive cervical cancer and high-grade squamous intraepithelial lesions classed as severe dysplasia. Other oncogenic types were closely associated with high-grade squamous intraepithelial lesions of moderate dysplasia and lowgrade squamous intraepithelial lesions. This suggests that risk for progression to invasion in squamous intraepithelial lesions is closely related to human papillomavirus genotype. Knowledge of the associated human papillomavirus type in women with morphologic squamous intraepithelial lesions may help to clarify risk for progression.

Modern Pathology (2007) 20, 167-174. doi:10.1038/modpathol.3800723; published online 22 December 2006
\end{abstract}

Keywords: cervical carcinoma; human papillomavirus; low-grade squamous intraepithelial lesion; high-grade squamous intraepithelial lesion; HPV genotyping; cervical carcinogenesis

Traditionally, cervical carcinogenesis was thought to evolve through a series of increasingly abnormal intraepithelial patterns (ie, mild-moderate-severe

Correspondence: Dr RE Zuna, MD, Department of Pathology, Biomedical Sciences Building Room 451, University of Oklahoma Health Sciences Center, 940 Stanton L. Young Building, Oklahoma City, OK 73104, USA.

E-mail: rosemary-zuna@ouhsc.edu

Received 6 April 2006; revised 4 October 2006; accepted 9 October 2006; published online 22 December 2006 dysplasia) followed, in a minority of cases, by acquisition of the ability to invade and metastasize. The identification of human papillomavirus as a causative agent in the spectrum of cervical lesions ${ }^{1-7}$ has added a high degree of complexity to understanding the carcinogenic process in that over 30 different HPV types have been identified in the spectrum of cervicovaginal lesions. Fifteen of these genotypes are widely considered to be oncogenic..$^{7-9}$ 
In recent years, evidence has accumulated to suggest that low-grade squamous intraepithelial lesions (LSIL) are typically self-limited, wart-like lesions that do not persist or progress to a more significant lesion. ${ }^{10,11}$ Since then, the Bethesda System $^{12,13}$ has implemented a biphasic categorization of squamous intraepithelial lesions, that is, lowgrade squamous intraepithelial lesions (LSIL) and high-grade squamous intraepithelial lesions (HSIL). The latter category combined moderate dysplasia (CIN2) and severe dysplasia/carcinoma in situ (CIN3) into a single HSIL category.

In a previous study, ${ }^{14}$ we compared the HPV types associated with HSIL and invasive cancer in order to identify a subset of HSIL lesions that are at increased risk for progression to carcinoma. HPV types that were found as the sole HPV type in invasive cancers were thought to be capable of inducing a fully evolved malignant lesion while high-grade lesions with other HPV types might persist without progressing or indeed might regress. We categorized the HPV types into three categories, high risk (HR) HPV types, intermediate risk (IR) HPV types and low risk (LR) HPV types that correspond to the relative association of the HPV genotypes with invasive cancer. This approach, using three categories of HPV types, was originally suggested by Lorincz et $a 1^{15,16}$ using a smaller number of HPV types and Southern blots. We found that the pattern of HPV types in severe dysplasia (HSIL-S) closely resembled that for invasive carcinoma. However, moderate dysplasia (HSIL-M) had a different pattern of genotypes, typically IR types. It became clear that it was important for us to identify the patterns of HPV types in our cases of LSIL in order to clarify the significance of our findings in HSIL-M.

This report documents our experience adding LSIL cases to an expanded case list of HSIL and invasive cancer cases and provides the framework for our categorization of SIL and risk for invasion based upon morphology and HPV genotype.

\section{Materials and methods}

\section{Patient Samples}

This study was approved by the Institutional Review Board at the University of Oklahoma Health Sciences Center. The acquisition and handling of patient samples in this study have been described previously. ${ }^{14}$ Briefly, ThinPrep ${ }^{\circledR}$ Pap tests from the OU Medical Center Cytopathology service with cytologic interpretations of squamous intraepithelial lesions (SIL) or carcinoma were identified after sign-out. The residual PreservCyt ${ }^{\circledR}$ sample (Cytyc Corporation, Boxborough, MA) was used for HPV genotyping using the reverse line blot method (Roche Molecular Systems, Alameda, CA). ${ }^{14}$ The ThinPrep ${ }^{\circledR}$ Pap slides from the SIL cases were coded, and independently reviewed in a blinded fashion and without knowledge of the HPV results by two cytopathologists (RZ and YL or RM). Negative cases were also included in order to preclude bias. While adhering to the overall Bethesda System categorization in our laboratory, we also retain the traditional 3-category terminology for SIL lesions, that is, dysplasia ${ }^{17}$ and CIN. ${ }^{18}$ Thus, we have diagnostic categories of HSIL-moderate dysplasia (CIN2) and HSIL-severe dysplasia (CIN3) that we designate HSIL-M and HSIL-S, respectively. The criteria for HSIL, including HSIL-M and HSIL-S, that are used in our laboratory have been previously described. ${ }^{14}$ Briefly, HSIL is characterized by dysplastic cells with dense, round-oval cytoplasm and increased nuclear-cytoplasmic ratio. In general, the cytoplasmic characteristics of HSIL are those of metaplastic cells, except in keratinizing dysplasias in which the categorization is based upon nuclear/cytoplasmic ratio. In severe dysplasia (HSIL-S) (Figure 1a), the cell size is small (comparable to parabasal cells) and the nuclear-cytoplasmic ratio is exaggerated with the nucleus occupying greater than half of the cytoplasmic diameter. HSIL cells with larger amounts of dense cytoplasm are categorized as moderate dysplasia (HSIL-M) (Figure 1b). Cells diagnostic for LSIL had enlarged atypical nuclei greater than three times normal nuclear size ${ }^{12}$ set in abundant, thin, polygonal cytoplasm that is typical for superficial squamous cells (Figure 1c) and/or HPV cytopathic effect as defined by Bethesda $2001 .^{13}$ If more than one diagnostic category was recognized, the cases were assigned to the highest grade.

The cytologic categories were assigned values from 1 to $5^{14}$ as follows: $1=$ negative; $2=$ ASCUS (atypical cell of undetermined significance); $3=$ mild dysplasia (CIN I, LSIL); $4=$ moderate dysplasia (CIN II; HSIL-M); $5=$ severe dysplasia/ carcinoma in situ (CIN III; HSIL-S). When mixed SIL patterns were identified, the cases were categorized by the most severe cell type identified. The scores of the two independent pathologists and the corresponding score of the original clinical interpretation made by one of four rotating diagnostic cytopathologists were summed for each case to derive a final score that represented a consensus diagnosis. Final scores of 3-4 were considered to be negative; $5-7$, atypical (ASCUS); 8-10, mild dysplasia (LSIL); 1113, moderate dysplasia (HSIL-M); and 14-15, severe dysplasia/carcinoma in situ (HSIL-S). In this report, all new cytology cases of SIL were categorized according to the consensus interpretation and added to our earlier cases. ${ }^{14}$

Unlike the SIL cases, all cervical cancers had histologic diagnoses and were entered into the study on the basis of the tissue diagnosis. As most of our cancer cases were referred to our institution with a diagnosis of carcinoma, few cases have routine Pap tests in our laboratory. We felt that it was important to characterize the genotypes found in actual cancers as a means of verifying the carcinogenic HPV types in our population. We, therefore, col- 

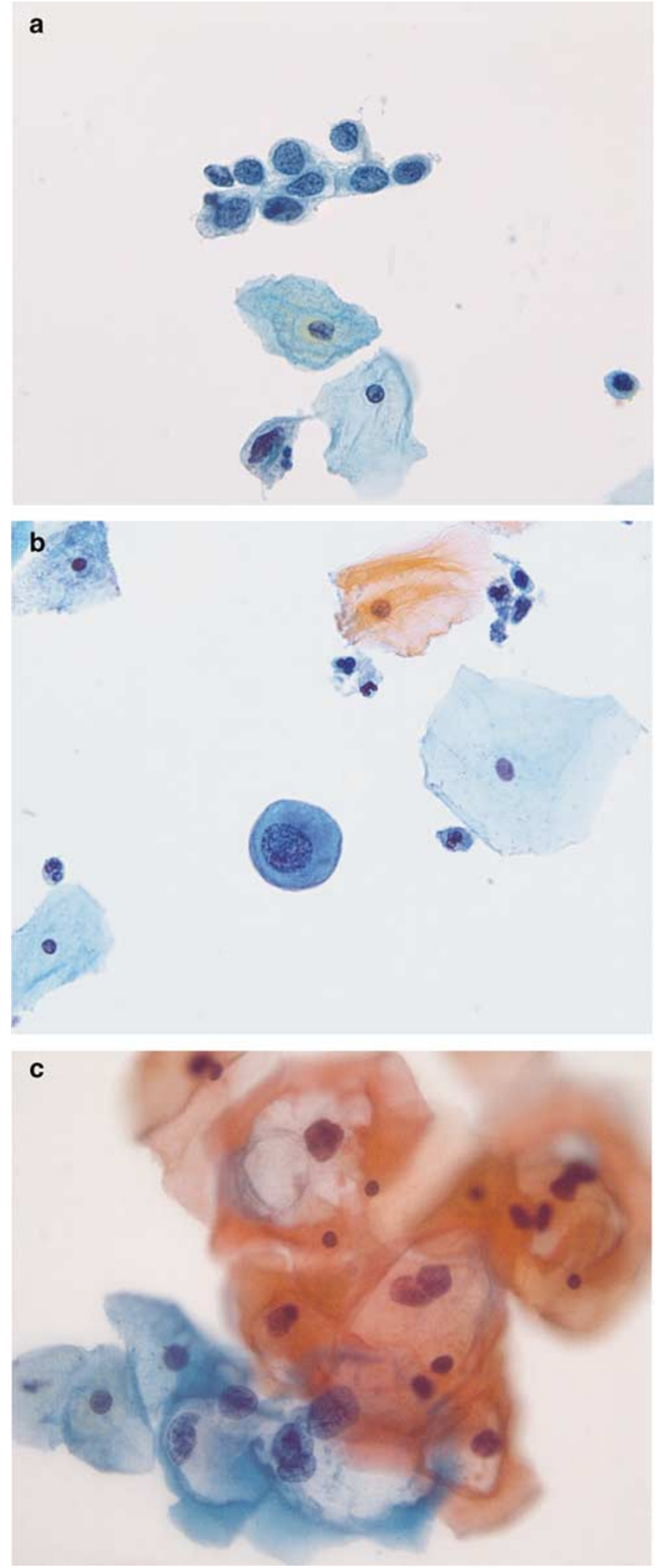

Figure 1 Examples of cytologic patterns as defined in this study: (a) HSIL-S: 29-year-old Native American woman; HPV 16 was found in this sample (modified Papanicolaou stain, $\times 60$, original magnification). (b) HSIL-M: 45-year-old woman; HPV 51 was the single genotype identified (modified Papanicolaou stain, $\times 60$, original magnification). (c) LSIL with koilocytotic halos; 32-yearold woman; HPV 66 was the only HPV identified (modified Papanicolaou stain, $\times 60$, original magnification). lected cytologic samples from unfixed cervical cancer tissues received in the Surgical Pathology Laboratory by brushing the cut surface with a Cytobrush $^{\circledR}$ (MedScand AB, Malmö, Sweden) and dislodging the cells into PreservCyt ${ }^{\circledR}$. These samples were then sent to the Molecular Pathology Laboratory and handled identically with those retrieved from the diagnostic Cytopathology Laboratory.

The histologic diagnosis and cell type for each of 93 cases of invasive cervical carcinoma were originally made by a pathologist on the OU Medical Center diagnostic Surgical Pathology service, independently verified (RZ) without knowledge of the HPV type, and categorized according to the WHO classification. ${ }^{19}$ There were 75 squamous cell carcinomas, nine adenocarcinomas, seven adenosquamous carcinomas, and two poorly differentiated carcinomas. Of the 282 cases reported here, 102 were included ${ }^{14}$ in our earlier communication.

\section{HPV Genotyping}

DNA from $5 \mathrm{ml}$ aliquots of residual PreservCyt ${ }^{\circledR}$ sample was isolated as previously described. ${ }^{14}$ For each specimen, $10 \mu \mathrm{l}$ of extracted DNA was subject to PCR amplification using the HPV consensus PCR and genotyping method (Roche Molecular Systems Inc., Alameda, CA), as indicated by the manufacturer. Briefly, this method ${ }^{20,21}$ utilizes biotinylated primers (PGMY09/PGMY11) that amplify a $450 \mathrm{bp}$ fragment of the L1 open reading frame of 27 genital HPV genotypes. A control set of biotinylated primers simultaneously amplifies a $268 \mathrm{bp}$ fragment of the human $\beta$-globin gene in each reaction. Resulting PCR products were then denatured and hybridized to separate HPV genotyping strips containing an immobilized array of 27 different HPV probes. These probes were categorized as high risk: 16, 18, 31, 45; intermediate risk: 33, 35, 39, 51, 52, 56, 58, 59, 68 [ME180], 73 [MM9, P238A], 82 [MM4, W13B], 83 [MM7, P291]; and low risk: 6, 11, 26, 40, 42, 53, 54, 55, 57, 66, 84 [MM8, P155]. Hybridized PCR products were visualized by using a streptavidin-horseradish peroxidase detection system. Each genotyping strip contains $\beta$-globin probes at two different concentrations to control for sample inhibition and sample sufficiency. A negative control sample was included with each batch of specimens tested and was consistently negative. Two cases failed to amplify either $\beta$-globin or any HPV sequence and were excluded from this study.

\section{Statistical Analyses}

Statistical analyses were performed using Systat Version 10 (Systat Software Inc., Richmond, CA) and StatXact 5 (Cytel Software Inc., Cambridge, MA). Inferential statistics used for tabular data included Fisher's exact tests, Pearson $\chi^{2}$, Odds ratios with 
95\% confidence intervals, and Cochran-Armitage linear trend tests. All $P$-values were two-sided. Statistical significance was ascribed to $P \leq 0.05$.

\section{Results}

The 282 patient samples constitute an unselected population of women with prevalent SIL or cervical carcinoma. Overall, our patients are a high-risk population for the development of cervical neoplasia with varying histories of previous Pap screening. The median age and age range for each of the final diagnostic categories were as follows: LSIL: 23 years (range 15-49 years); HSIL-M: 26 years (range: 1678); HSIL-S: 32 years (range: 17-66) and invasive cancers: 43 years (range 22-76).

HPV genotyping results were initially grouped according to HPV risk group and diagnostic category as shown in Table 1. In total, 456 individual HPV types were identified in the 282 women in this study. In all, 12 cases (4.2\%) with satisfactory amplification of the $\beta$-globin fragment had negative HPV results. Overall, 270 cases $(95.7 \%)$ harbored at least one HPV genotype. These included 63 (94.4\%) LSIL cases, 57 (98.3\%) HSIL-M, 63 (98.4\%) HSIL-S and $87(93.5 \%)$ cancers. Two or more HPV types (up to six types in a single case) were identified in 109 $(38.7 \%)$ cases studied. As previously reported, ${ }^{14}$ the HR HPV types continued to be the dominant group in both HSIL-S $(49.1 \%)$ and invasive carcinoma
(77.4\%) when all HPV types were included. Similarly, the dominant role of IR viral types $(52.2 \%)$ in HSIL-M continued. LSIL harbored the largest percentage $(36.8 \%)$ of LR genotypes but IR HPV types were more frequent $(45.1 \%)$ in that category as well. LSIL showed the highest percentage of multiple infections ( $56.7 \%$ or 38 cases); however, the differences between the number of multiple infections among the intraepithelial lesions was not significant $\left(\chi^{2}=4.7 ; \mathrm{df}=4 ; P=0.32\right)$.

A similar distribution was seen when each case was categorized according to the single highest risk HPV type present (Table 2). Overall, the association of highest risk HPV category with diagnostic category was highly significant for the 282 women $\left(\chi^{2}\right.$ test for independence: $\chi^{2}=104.9 ; \mathrm{df}=9$; $P<0.0001)$. While HSIL cases were associated with a dominant HPV category (HR for HSIL-S and IR for HSIL-M), the pattern for LSIL was biphasic (LR and IR). $\chi^{2}$ analysis for independence showed significant differences in the distribution of the HPV categories in LSIL compared with HSIL-M $\left(\chi^{2}=14.63\right.$; $\mathrm{df}=3$; $P=0.002$ ) and in the distribution of HSIL-M compared with HSIL-S $\left(\chi^{2}=16.44 ; \mathrm{df}=3 ; P=0.0009\right)$ but not that of HSIL-S compared with invasive carcinoma $\left(\chi^{2}=5.2 ; \mathrm{df}=2 ; P=0.073\right)$. These results (Figure 2) suggest that there is an association of morphologic pattern with category of HPV type such that three major categories of SIL lesions can be discerned, that is, HSIL-S with HR viruses, HSIL-M and many LSIL cases with IR HPV types and LSIL

Table 1 Distribution of 456 individual HPV types in 282 cases according to HPV risk group and diagnostic category

\begin{tabular}{|c|c|c|c|c|c|}
\hline \multirow[t]{2}{*}{ HPV category } & \multicolumn{5}{|c|}{ Diagnostic category } \\
\hline & $\operatorname{LSILN} N(\%)^{\mathrm{a}}$ & $H S I L-M N(\%)$ & $H S I L-S N(\%)$ & Invasive carcinoma $N(\%)$ & Total HPV N (\%) \\
\hline High risk & $24(18.0)$ & $29(26.1)$ & 55 (49.1) & $80(80.0)$ & $188(41.2)$ \\
\hline Intermediate risk & $60(45.1)$ & $58(52.2)$ & 38 (33.9) & $17(17.0)$ & $173(37.9)$ \\
\hline Low risk & $49(36.8)$ & $24(21.6)$ & $19(17.0)$ & $3(3.0)$ & $95(20.8)$ \\
\hline Total HPV & $133(100)$ & $111(100)$ & $112(100)$ & $100(100)$ & $456(100)$ \\
\hline
\end{tabular}

${ }^{\mathrm{a}} \mathrm{N}=$ number of HPV genotypes identified in each diagnostic category. Percentage was defined by the number of individual HPV types divided by the total number of HPV identified in each column (diagnostic category). The percentages were calculated down the column.

Table 2 Distribution of 282 cervical cases according to the single highest risk HPV type present and diagnostic category

\begin{tabular}{|c|c|c|c|c|c|}
\hline HPV category & $\operatorname{LSILN} N(\%)^{\mathrm{a}}$ & $H S I L-M N(\%)$ & $H S I L-S N(\%)$ & Invasive carcinoma $N(\%)$ & Total HPV N (\%) \\
\hline High risk & $18(26.9)$ & $25(43.1)$ & $50(78.1)$ & $78(83.8)$ & $171(60.6)$ \\
\hline Intermediate risk & $30(44.8)$ & $31(53.5)$ & $13(20.3)$ & $9(9.7)$ & $83(29.4)$ \\
\hline Low risk & $15(22.4)$ & $1(1.7)$ & 0 & 0 & $16(5.7)$ \\
\hline No HPV & $4(6.0)$ & $1(1.7)$ & $1(1.6)$ & $6(6.5)$ & $12(4.2)$ \\
\hline Total & 67 (100) & $58(100)$ & $64(100)$ & $93(100)$ & $282(100)$ \\
\hline Multiple HPV & $38(56.7)^{\mathrm{b}}$ & $31(53.4)$ & $30(46.9)$ & $10(9.1)$ & $109(38.7)$ \\
\hline
\end{tabular}

\footnotetext{
${ }^{\mathrm{a}} \mathrm{N}=$ number of cases in each diagnostic category sorted according to the single highest risk HPV type identified. The percentage is calculated down the row with the total number of cases used as the denominator.

${ }^{\mathrm{b}}$ Percent is calculated on the total number of cases in the column.
} 
cases that harbor LR viruses only. Similarly, there was a linear trend in the association of HR HPV with increasing severity of diagnostic category (Cochran-Armitage trend test: $P<0.0001$ ). In addition, the pattern of HPV types in HSIL-S and invasive carcinomas were comparable and reinforced the accepted role of HSIL-S as the immediate precursor to invasive carcinoma. At the same time, the differences in HPV types found in LSIL and HSIL-M compared with carcinoma suggest that these lesions have limited risk for progression to malignancy.

Table 3 shows the distribution of the 456 individual HPV types found in the spectrum of cervical lesions included in this study. While HR HPV 16 (54.8\%) and HPV 18 (24.7\%) were the most frequent HPV types identified in invasive cancers, the distribution patterns of HPV types in the intraepithelial lesions was highly varied. HSIL-S also showed HPV 16 as the most frequent HPV type present $(65.6 \%)$. The next most frequent HPV types in HSIL-S were IR HPV 52 (14.1\%), IR HPV 39 $(12.5 \%)$, and HR HPV 18 (10.9\%). For HSIL-M and
LSIL, there was a wide range of HPV types with no single dominant genotype. For HSIL-M, HR HPV16 and IR HPV 51 were each present in $19.0 \%$ of cases,

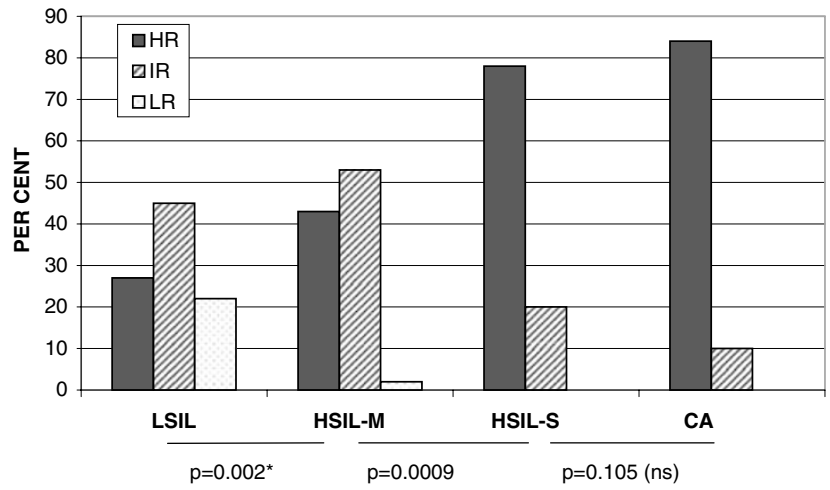

${ }^{*}$ chi square for independence

Figure 2 This graph shows the distribution of 282 individual women according to HPV category and diagnostic category. Each woman is represented once and categorized according to the most abnormal cells in her sample and according to the highest risk HPV type identified.

Table 3 Distribution of 456 individual HPV types in 282 cervical lesions

\begin{tabular}{|c|c|c|c|c|c|c|}
\hline HPV category & HPV type & $\operatorname{LSIL~} N(\%)^{\mathrm{a}}$ & $H S I L-M N(\%)$ & HSIL-S N (\%) & Carcinoma $N(\%)^{a}$ & Total HPV N $(\%)$ \\
\hline \multirow[t]{4}{*}{ High risk } & 16 & $10(14.9)$ & $11(19.0)$ & $42(65.6)$ & $52(55.9)^{\mathrm{b}}$ & $115(40.7)$ \\
\hline & 18 & $5(7.5)$ & $6(10.3)$ & $7(10.9)$ & $23(24.7)^{\mathrm{b}}$ & 41 (14.5) \\
\hline & 31 & $6(9.0)$ & $8(13.8)$ & $4(6.2)$ & $1(1.1)^{b}$ & $19(6.7)$ \\
\hline & 45 & $3(4.5)$ & $4(6.9)$ & $2(3.1)$ & $4(4.3)^{\mathrm{b}}$ & $13(4.6)$ \\
\hline \multirow[t]{12}{*}{ Intermediate risk } & 33 & $1(1.5)$ & $4(6.9)$ & $2(3.1)$ & $3(3.2)^{\mathrm{b}}$ & $10(3.5)$ \\
\hline & 35 & $3(4.5)$ & $8(13.8)$ & $6(9.4)$ & $3(3.2)^{\mathrm{b}}$ & $20(7.1)$ \\
\hline & 39 & $8(11.9)$ & 7 (12.1) & $8(12.5)$ & $1(1.1)^{\mathrm{b}}$ & $24(8.5)$ \\
\hline & 51 & 13 (19.4) & $11(19.0)$ & $2(3.1)$ & $1(1.1)$ & $27(9.6)$ \\
\hline & 52 & $6(9.0)$ & 9 (15.5) & $9(14.1)$ & 1 (1.1) & $25(8.9)$ \\
\hline & 56 & $10(14.9)$ & $3(5.2)$ & $3(4.7)$ & $1(1.1)$ & $17(6.0)$ \\
\hline & 58 & $5(7.5)$ & $4(6.9)$ & $3(4.7)$ & 0 & $12(4.2)$ \\
\hline & 59 & $5(7.5)$ & 7 (12.1) & $2(3.1)$ & $2(2.2)$ & $16(5.7)$ \\
\hline & 68 & $3(4.5)$ & $1(1.7)$ & $1(1.6)$ & $1(1.1)^{\mathrm{b}}$ & $6(2.1)$ \\
\hline & 82 & $2(3.0)$ & 0 & 0 & $1(1.1)^{b}$ & $3(1.0)$ \\
\hline & 83 & $1(1.5)$ & $2(3.4)$ & $1(1.6)$ & $1(1.1)^{b}$ & $5(1.8)$ \\
\hline & 73 & $3(4.5)$ & $2(3.4)$ & $1(1.6)$ & $2(2.2)$ & $8(2.8)$ \\
\hline \multirow[t]{12}{*}{ Low risk } & 6 & $9(13.4)$ & $1(1.7)$ & $1(1.6)$ & 0 & $11(3.9)$ \\
\hline & 11 & $4(6.0)$ & 0 & $1(1.6)$ & 0 & $5(1.8)$ \\
\hline & 26 & 0 & 0 & $1(1.6)$ & 0 & $1(0.3)$ \\
\hline & 40 & $1(1.5)$ & $1(1.7)$ & 0 & 0 & $2(0.7)$ \\
\hline & 42 & $2(3.0)$ & 0 & $1(1.6)$ & 0 & $3(1.1)$ \\
\hline & 43 & 0 & 0 & 0 & 0 & 0 \\
\hline & 44 & 0 & 0 & 0 & 0 & 0 \\
\hline & 53 & $11(16.4)$ & 7 (12.1) & $4(6.2)$ & 0 & $22(7.8)$ \\
\hline & 54 & $6(9.0)$ & $3(5.2)$ & $2(3.1)$ & $2(2.2)$ & $13(4.6)$ \\
\hline & 55 & $3(4.5)$ & $3(5.2)$ & $3(4.7)$ & $1(1.1)$ & $10(3.5)$ \\
\hline & 66 & 11 (16.4) & $4(6.9)$ & $3(4.7)$ & 0 & $18(6.4)$ \\
\hline & 84 & $2(3.0)$ & $5(8.6)$ & $3(4.7)$ & 0 & $10(3.5)$ \\
\hline HPV negative & & $4^{\mathrm{c}}(6.0)$ & $1(1.7)$ & $1(1.6)$ & $6(6.5)$ & $12(4.3)$ \\
\hline Total HPV & & 133 & 111 & 112 & 100 & 456 \\
\hline Total cases & & 67 & 58 & 64 & 93 & 282 \\
\hline
\end{tabular}

${ }^{\text {a }}$ Percentages were based upon number of cases but because of multiple HPV types in many cases, the column percentages do not add to $100 \%$.

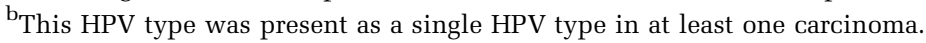

${ }^{\mathrm{c}}$ Two out of four cases subsequently tested positive for other HPV types not included in this version of the reverse line blot test (data not shown). 
while IR HPV 52 (15.5\%), HR HPV 31 (13.8\%), IR HPV $35(13.8 \%)$ were also frequently present. The most frequent HPV type found in LSIL was IR HPV $51(19.4 \%)$ followed by IR HPV 53 and LR HPV 66 (16.4\% each), HR HPV 16 and IR HPV 56 (14.9\% each).

A single HPV type was identified in $76(81.7 \%)$ cancers in this series. These included HR HPV 16 (47/76 cases or $68.1 \%$ ), HR HPV 18 (16 or $21.0 \%$ ), HR HPV 45, IR HPV 33 and IR HPV 35 (two cases or $2.6 \%$ for each). HR HPV 31, IR HPV 39, IR HPV 68, IR HPV 73 and IR HPV 83 were each present in one case as a single HPV type (1.3\% each). Notably, LR HPV 66, elsewhere considered to be oncogenic, ${ }^{8,9}$ was a frequent genotype in LSIL in this series (Figure 1c) but was not found in any of our cases of carcinoma (Table 3).

While approximately half of HPV $16(51 / 114$ or $45.6 \%)$ and HPV 18 (56.1\%) genotypes identified in this series were found in invasive cancers (see Table 3 ), other HPV types that are generally categorized as oncogenic were found more commonly in intraepithelial lesions. For instance, of the 24 total cases with HPV 39 in this series, only one $(4.2 \%)$ was found in a cancer while the remaining HPV 39 cases were intraepithelial lesions evenly distributed among the SIL categories (Table 3). Similarly, $3.7 \%$ of HPV 51 ( 1 of 27 ) and $4.0 \%$ of HPV 52 ( 1 of $25)$ genomes were identified in cancer cases, in contrast to the large majority found in intraepithelial lesions.

\section{Discussion}

Several large international meta-analyses have tabulated the HPV types associated with cervical cancers. ${ }^{8,22,23}$ A recent conference sponsored by the International Agency for Research Against Cancer $\left(\right.$ IARC) ${ }^{9}$ concluded that 13 HPV types are carcinogenic to humans, including HPV 16, 18, 31, $33,35,39,45,51,52,56,58,59$ and 66 . In our studies, which compared the HPV genotypes in cancer with those in intraepithelial lesions, a limited number of HPV genotypes were found as the sole HPV in cervical cancers. These included HPV 16, 18, 31, 45, 33, 35, 39, 68, 73 and 83 although additional HPV types were associated with HSIL cases. Only HPV 16 and HPV 18 were increased in cancers relative to lesser grade categories. Most of the remaining HPV types cited as carcinogenic in the IARC listing were found predominantly in intraepithelial lesions in our series, with only a small number found in cancers. Interestingly, a meta-analysis of published studies performed by Clifford et al ${ }^{24}$ compared HPV types preferentially associated with high-grade intraepithelial lesions and cancers and found that only HPV 16, 18, and 45 were more prevalent in cancers than in intraepithelial lesions while the reverse was true for HPV 31, 33, 52 and 58.
In our studies using a sensitive PCR technique that allows identification of multiple genotypes in a single reaction, HR HPV genotype, especially HPV 16 and 18, were also strongly associated with invasive cancer and HSIL. This association was not found for the other HPV types (categorized here as IR) that are widely held to be oncogenic. The latter genotypes were frequently present in intraepithelial lesions but only occasionally found as a single type in cancers. This suggests that there are undefined differences in the transformationrelated changes associated with HPV types other than HPV 16 or 18 such that only a limited number of lesions with these IR HPV types will develop progressive disease. Clinically, this becomes important when considering the genotypes to be included in preventive vaccines and also when planning the appropriate follow-up of women with SIL. This issue is particularly important for young women who plan future childbearing. Recent studies have shown that women who have had cervical LEEP procedures are at increased risk for premature rupture of membranes and preterm delivery. ${ }^{25,26}$

Our use of moderate dysplasia (HSIL-M) as a discrete cytologic category in this study may be controversial. Our initial approach was to follow the Bethesda categories and to combine moderate and severe dysplasia into a single HSIL category. However, when we queried our original data set comparing HSIL and carcinoma according to subgroup of HSIL, the dichotomy between the HPV genotypes in HSIL-M and HSIL-S emerged (see Figure 2). ${ }^{14}$ This pattern persisted with the addition of new cancer and HSIL cases and inclusion of LSIL in this report. In the current study, we were surprised to find that the pattern of genotypes found in HSIL-M more closely resembled that of LSIL than HSIL-S and cancer. This finding could relate to poor reproducibility of HSIL-M (cytology) and CIN2 (histology) among pathologists and a lack of consensus for morphologic criteria. ${ }^{27,28}$ Indeed, cytologic criteria for HSIL-M have not been defined by the Bethesda System. However, our results suggest that HSIL is a heterogeneous category in terms of biological potential and that HPV genotyping can aid in assessing the risk for progression. As HPV genotype is an objective parameter, the combined approach of HPV genotyping and cytologic interpretation is likely to be similarly effective for laboratories that follow current Bethesda guidelines and combine HSIL-M and HSIL-S into a single category. Therefore, we do not believe that HSIL need be subdivided in the routine diagnostic setting for laboratories who have abandoned that approach.

The results of this study suggest that three major categories of SIL lesions can be discerned based upon the category of HPV type (as defined here) present in the cytologic samples: (1) SIL, typically HSIL, associated with HR HPV types that appear to be at increased risk for progression to carcinoma; (2) SIL (typically LSIL and HSIL-M) associated with IR 
HPV types with limited or indeterminate risk for progression; (3) LSIL associated with LR HPV types with little or no risk for progression. If this pattern is verified by others, it is possible that these groups can be triaged into different follow-up patterns that correlate with risk for progression to cancer. The biological potential of LSIL with HR HPV types is unclear from our data but such cases would appear to warrant close follow-up. This is because data from the ALTS Trial ${ }^{29}$ found that ASCUS and LSIL cases with HPV 16 at baseline had a 2-year cumulative absolute risk of 32.5 and $39.1 \%$, respectively for the diagnosis of CIN3 or higher within 2 years. Thus, despite the low-risk morphologic pattern, the risk for the development of HSIL-S and cancer appears to be related to HR HPV status.

The significance of these observations could be far reaching in that women harboring IR HPV types are now considered to have oncogenic HPV according to current practice. When HPV testing is performed for ASC-US cytology or as part of screening in women over the age of 30 years, these women will have positive tests for oncogenic HPV (as defined by the current probe sets) and are referred for colposcopy. They may be identified with moderate dysplasia (CIN2) and referred for an excisional procedure, typically LEEP. However, validation of our results with clinical studies could permit a more conservative approach for many of these women. Similarly, testing for a smaller number of truly oncogenic HPV types either alone or as a second tier to a broadspectrum HPV test might generate considerable savings of health care resources if conservative management could be justified for more patients. Khan et al. ${ }^{30}$ have proposed specific genotyping for HPV 16 and 18 in women who are HPV positive, with a more conservative follow-up algorithm for women who are HPV positive for oncogenic HPV types other than HPV16 or 18. Such an approach would identify the women in our HSIL-M category who should be followed-up like those with HSIL-S. Similarly, persistent HSIL morphology with positive oncogenic HPV test and negative HPV16/HPV 18 tests would trigger intervention in women with IR genotypes who may be at risk for progression. Finally, the morphologic spectrum associated with most IR and HR HPV types suggests that cytologic assessment remains an important component in the identification and clinical management of women with HPV-associated lesions.

\section{Acknowledgements}

We acknowledge the assistance of Drs Michael Gold, Scott McMeekin, Joan Walker, Natalie Gould, Todd Tillmanns, Teresa Rutledge, Barbara Bane, Zoltan Laszik, Gregory Blakey, Chimen Kesserwan, Adeboye Osunkoya, Ildiko Nagy, Hans Iwenofu, W Grant Davis, Pablo Souza, Fred Bierbaum, Robyn
Potts, Jane Zhou, and Ravindranauth Sawh for their assistance in acquiring samples for this study. We also thank Cora Wright, Patricia Parrish, Danita Young in the Cytopathology Laboratory as well as Patricia Eneff and Jewel Uselton in the Surgical Pathology Laboratory of OU Medical Center for their enthusiastic help in specimen handling. In addition, we are grateful to Roche Molecular Systems, Alameda, CA for the gift of the HPV consensus PCR and genotyping reagents used in this study.

\section{References}

1 zur Hausen H. Human papilloma viruses and their possible role in squamous cell carcinomas. Curr Top Microbiol Immunol 1977;78:1-3.

2 Meisels A, Fortin R. Condylomatous lesions of the cervix and vagina. I. Cytologic patterns. Acta Cytol 1976;20:505-509.

3 Purola E, Savia E. Cytology of gynecologic condyloma acuminatum. Acta Cytol 1977;21:26-31.

4 Meisels A, Fortin R, Roy M. Condylomatous lesions of the cervix. II. Cytologic, colposcopic and histopathologic study. Acta Cytol 1977;21:379-390.

5 Schiffman MH, Bauer HM, Hoover RN, et al. Epidemiologic evidence showing that human papillomavirus infection causes most cervical intraepithelial neoplasia. J Natl Cancer Inst 1993;85:958-964.

6 Walboomers JMM, Jacobs MV, Manos MM, et al. Human papillomavirus is a necessary cause of invasive cervical cancer worldwide. J Pathol 1999;189:12-19.

7 Bosch FX, Lorincz A, Muñoz N, et al. The causal relation between human papillomavirus and cervical cancer. J Clin Pathol 2002;55:244-265.

8 Muñoz N, Bosch FX, de Sanjosé S, et al, International Agency for Research on Cancer Multicenter Study Group. Epidemiologic classification of human papillomavirus types associated with cervical cancer. N Engl J Med 2003;348:518-527.

9 Cogliano V, Baan R, Straif K, et al, WHO International Agency for Research on Cancer. Carcinogenicity of human papillomaviruses. Lancet Oncol 2005;6:204.

10 Kiviat NB, Critchlow CW, Kurman RJ. Reassessment of the morphological continuum of cervical intraepithelial lesions: Does it reflect different stages in the progression to cervical carcinoma? In: Muñoz N, Bosch FX, Shah KV, Meheus A (eds). The Epidemiology of Cervical Cancer and Human Papillomavirus. International Agency for Research on Cancer: Lyon, 1992, pp 59-66.

11 Kiviat NB. Natural history of cervical neoplasia: overview and update. Am J Obstet Gynecol 1996;175: 1099-1104.

12 Kurman R, Solomon DS. The Bethesda System for Reporting Cervical/vaginal Cytologic Diagnoses. Springer-Verlag: New York, 1993.

13 Solomon D, Davey D, Kurman R, et al. The 2001 Bethesda system: terminology for reporting results of cervical cytology. JAMA 2002;287:2114-2119.

14 Zuna RE, Allen R, Moore WM, et al. Comparison of human papillomavirus genotypes in high-grade squamous intraepithelial lesions and invasive cervical carcinoma: evidence for differences in biologic 
potential of precursor lesions. Mod Pathol 2004;17: 1314-1322 advance published online July 16, 2004; doi:10.1038/modpathol.3800223.

15 Lorincz A, Temple GF, Kurman RJ, et al. Oncogenic association of specific human papillomavirus types in cervical neoplasia. J Natl Cancer Inst 1987;79:671-677.

16 Lorincz AT, Reid R, Jenson AB, et al. Human papillomavirus infection of the cervix: relative risk associations of 15 common anogenital types. Obstet Gynecol 1992;79:328-337.

17 Patten SF. Diagnostic Cytology of the Uterine Cervix, 2nd edn. S Karger: Basel, 1978.

18 Richart R. The natural history of cervical intraepithelial neoplasia. Clin Obstet Gynecol 1967;10: 748-784.

19 Scully RE, Bonfiglio TA, Kurman RJ (eds). Classification of Female Genital Tract Tumors, 2nd edn. Springer-Verlag: Berlin, 1994.

20 Gravitt PE, Peyton CL, Apple RJ, et al. Genotyping of 27 human papillomavirus types by using L1 consensus PCR products by a single-hybridization, reverse line blot detection method. J Clin Microbiol 1998;36:3020-3027.

21 Gravitt PE, Peyton CL, Alessi TQ, et al. Improved amplification of genital human papillomaviruses. J Clin Microbiol 2000;38:357-361.

22 Bosch FX, Manos MM, Muñoz N, et al. Prevalence of human papillomavirus in cervical cancer: a worldwide perspective. J Natl Cancer Inst 1995;87:796-802.

23 Clifford GM, Smith JS, Plummer M, et al. Human papillomavirus types in invasive cervical cancer worldwide: a meta-analysis. Br J Cancer 2003;88: 63-73.

24 Clifford GM, Smith JS, et al. Comparison of HPV type distribution in high grade lesions and cervical cancer: a meta-analysis. Br J Cancer 2003;89:101-105.

25 Sadler L, Saftlas A, Wang W, et al. Treatment for cervical intraepithelial neoplasia and risk of preterm delivery. JAMA 2004;291:2100-2106.

26 Samson SL, Bentley JR, Fahey TJ, et al. The effect of loop electrosurgical excision procedure on future pregnancy outcome. Obstet Gynecol 2005;105: $325-332$.

27 Confortini M, Biggeri A, Cariaggi MP, et al. Intralaboratory reproducibility in cervical cytology. Results of the application of a 100-slide set. Acta Cytol 1993;37:49-54

28 Stoler MH, Schiffman M. Interobserver reproducibility of cervical cytologic and histologic interpretations: Realistic estimates form the ASCUS-LSIL Triage Study. JAMA 2001;285:1500-1505.

29 Castle PE, Solomon D, Schiffman M, et al. Human papillomavirus type 16 infections and 2 year absolute risk of cervical precancer in women with equivocal or mild cytologic abnormalities. J Natl Cancer Inst 2005;97:1066-1071.

30 Khan MJ, Castle PE, Lorincz AT, et al. The elevated 10 year risk of cervical precancer and cancer in women with human papillomavirus (HPV) type 16 or 18 and the possible utility of type-specific HPV testing in clinical practice. J Natl Cancer Inst 2005;97: 1072-1079. 